\title{
The artery of Adamkiewicz: More interesting than practical?
}

Despite major advancements in spinal cord protection during repairs of the thoracic aorta and thoracoabdominal aorta (TAAA), spinal cord injury (SCI) remains a devastating complication. Although the incidence of SCI has improved with adjuncts, the difficulty in eliminating SCI relates to a multitude of factors responsible for the preservation of the spinal cord blood supply. Critical factors pertinent to the integrity of the blood supply include hemodynamic status, technical aspects of the operation, adjuncts, neuromonitoring, pharmacologic agents used, and complications sustained during the intraoperative and postoperative course. It is the variability of all these factors that has made evaluation of SCI and its prevention difficult.

Tanaka and colleagues, ${ }^{1}$ authors of the Japanese Study of Spinal Cord Protection in Descending and Thoracoabdominal Aortic Repair (JASPAR) reported in this issue of the Journal, are to be commended for analyzing the significance of preoperative identification of the Adamkiewicz artery (AKA) during repairs of the descending thoracic and TAAA. In one of the largest multicenter registries for descending thoracic and TAAA repairs (2435 patients), Tanaka and colleagues ${ }^{1}$ identified the AKA in $88 \%$ ( $\mathrm{n}=1096$ ) of patients who had undergone preoperative radiographic assessment of the AKA ( $n=1252$ patients) for both open and endovascular repairs. The overall incidence of SCI, which was defined as a combination of "complete and incomplete," was commendable at $7 \%$ for the entire series, but with open repair was dependent on the extent of the aneurysm. The extents II and III TAAA were associated with the highest incidence at $14 \%$ each. Not surprisingly, in their assessment of SCI, Tanaka and colleagues, ${ }^{1}$ identified age, emergency presentation, and extent of repair (I and II) as risk factors for SCI. They were unable, however, to demonstrate preoperative AKA identification as a risk factor $(P=.299)$. This stands to reason, because identification of the AKA did not necessarily translate into what was done, as this information and the details on how and which intercostal arteries were reattached was not provided.

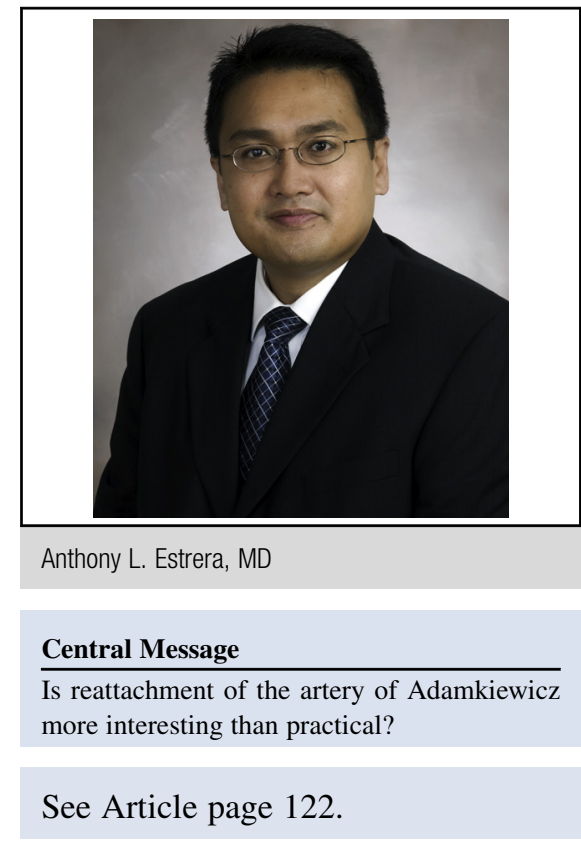

Appropriately, Tanaka and colleagues ${ }^{1}$ then analyzed a subgroup of patients who underwent repair of the TAAA in which the AKA was involved. It was this subgroup analysis that was most pertinent to the original aim of the study. As depicted in Figure 2 of Tanaka and colleagues, in the cases in which the aortic segment repaired involved the AKA, the AKA was "preserved or reattached" in 349 cases $(77 \%)$ and ligated or "closed" in $51(11 \%)$. Although the incidence of SCI in the ligated group was not reported, this group (closure of identified AKA without reconstruction) was associated with SCI $(P=.024$; Table 6 in the article of Tanaka and colleagues ${ }^{1}$ ). From this, Tanaka and colleagues $^{1}$ suggest a benefit of preoperative AKA identification with spinal cord protection.

Despite these data, it is unlikely that preoperative identification of the AKA will cause a change in how most surgeons deal with SCI during descending and TAAA repairs. The JASPAR registry confirmed that the AKA is most often ( $>90 \%$ of cases) associated with intercostal arteries $\mathrm{T} 8$ to $\mathrm{T} 12$. The importance of the reattachment of intercostal arteries $\mathrm{T} 8$ to $\mathrm{T} 12$, independent of the location of the AKA, has been previously established as protective against SCI. ${ }^{2}$ Many surgeons already consider reattachment of intercostal arteries T8 to T12 during TAAA repairs, and thus the preoperative identification of the AKA will alter little in the management of these repairs.

In summary, the JASPAR investigators are to be again commended for the data provided by this report. The 
information gleaned is as follows: (1) The report provides a contemporary view of open thoracic and TAAA repairs with respectable outcomes, including an overall mortality of $9 \%$ and an SCI rate of 7\%. (2) Preoperative radiographic identification of the AKA can be achieved in most cases with experienced centers. (3) The AKA arises from intercostal arteries T8 to T12 in most cases. (4) Although the preoperative identification of the AKA was not associated with SCI, the ligation of the intercostal arteries and AKA in the operated segment of the aorta was associated with SCI, suggesting a potential benefit to reattachment. Further study on factors that would allow better preoperative planning should be encouraged, but in the end this information becomes useful only if it changes our management.

\section{References}

1. Tanaka H, Ogino H, Minatoya K, Matsui Y, Higami T, Okabayashi H, et al. The impact of preoperative identification of the Adamkiewicz artery on spinal cord protection in descending and thoracoabdominal aortic repair. J Thorac Cardiovasc Surg. 2016;151:122-8.

2. Safi HJ, Miller CC III, Carr C, Iliopoulos DC, Dorsay DA, Baldwin JC. Importance of intercostal artery reattachment during thoracoabdominal aortic aneurysm repair. J Vasc Surg. 1998;27:58-66; discussion 66-8.

3. Estrera AL, Sheinbaum R, Miller CC III, Harrison R, Safi HJ. Neuromonitorguided repair of thoracoabdominal aortic aneurysms. J Thorac Cardiovasc Surg. 2010;140(6 Suppl):S131-5; discussion S142-6. 